\title{
IPTV 2.0 from Triple Play to Social TV
}

\author{
Hongguang Zhang, Hang Nguyen, Ioan Marius Bilasco, Gyu Myoung Lee, Hui Wang
}

\begin{abstract}
The great success of social technologies is transforming the Internet into a collaborative community. With a vision of IPTV 2.0, this paper presents our research work towards the exploitation of social phenomena in the domain of TV. Based on the advantage of IP Multimedia Subsystem (IMS) service architecture, the current IPTV service is extended from two aspects: TV-enriched communication and sociability-enhanced TV. Two applications namely TV Buddy and Social Electronic Program Guide (EPG) are proposed to demonstrate them respectively. Finally, we developed a prototype system on Ericsson IMS Software Development Studio (SDS).
\end{abstract}

Index Terms-IPTV, Service Deployment

\section{INTRODUCTION}

$\mathrm{I}_{\mathrm{d}}^{\mathrm{n}}$ nternet Protocol Television (IPTV) is a system where the digital television service is delivered using Internet Protocol [1]. The real power of IPTV is in the merging of entertainment and communication on an IP-based network, which provides significant opportunities to make the TV experience more interactive and personalized. For instance, Video on Demand (VoD) has made watching TV become an individual experience like surfing Internet. Another advantage of an IP-based network is the opportunity for convergence. This opportunity is amplified by IP Multimedia Subsystem (IMS) [2]. IMS-based IPTV has been extended to bundle with Internet access and Voice over Internet Protocol (VoIP) telecommunication services, which are sometimes referred to in marketing as "Triple Play" or even "Quadruple Play" [3] with the support of wireless. Under these trends, IMS-based IPTV technology has paved the way for a new class of interactive TV services: social TV. The social TV is a general term for technology that supports communication and interaction in the context of watching TV, or related to TV content. It can be seen as the application of social networking in the domain of TV. Specially, social TV allows users to share their TV experiences with others by providing a variety of ways for users to interact. For example, social TV may combine VoIP, Instant Message, Presence, and video conferencing etc. with TV. Moreover, social TV promises to change the role of passive viewers by turning them into active participants of the TV viewing

This work was supported in part by the ITEA-2 CAM4Home project.

Hongguang Zhang, Hang Nguyen, Gyu Myoung Lee, and Hui Wang are with the Institut Telecom SudParis, Evry, 91000, France (phone: +33 (0)1 6076 66 63; fax: +33 (0)1 607645 78; e-mail: hongguang.zhang@it-sudparis.eu).

Ioan Marius Bilasco is with the Laboratoire Informatique de Lille (LIFL). (e-mail: marius.bilasco@lifl.fr). experience, e.g. enhanced content navigation, user-generated content, social program bookmark etc.

Recently, the sociality has been explored in the domain of TV. AmigoTV [4] is a prototype implementation that combines broadcast television with rich communication and community support in order to leverage a rich social experience, and can been seen as a perfect example of where experience design can lead us to. CollaboraTV [5] supports asynchronous annotation of shows, currently limited to text. These comments can be generated at any point while watching a show. All future viewers will see these comments at the equivalent time they are created, and can leave their own. ConnecTV [6] is a service that enhances watching TV with sociability aspects. The pilot showed great value for operators and other parties that consider introducing sociability-enhancing services for their customers. However, the current solutions are essentially vendor-specific, which are neither scalable nor extensible. Furthermore, with the development of new technologies (e.g., Peer-to-Peer, Internet, and mobile network), the variety of video distribution has been increasing, which results in the multiple sources of video across different technical domains. The recent trend driven by end user is media mashup where service elements coming from more than one source are combined into an integrated user experience [7]. However, the characteristic of cross-domain aggregation is ignored by the current IPTV systems.

With a vision of IPTV 2.0, this paper presents our research work towards the exploitation of IMS, IPTV, social networking and multimedia metadata for the implementation of social TV. Our work focuses on enriching TV experiences by seamlessly integrating Web2.0 style from various technology domains. Social phenomenon can be utilized not only to enrich communication between viewers but also to enhance TV watching experiences. We extend the current IPTV service from two aspects: TV-enriched communication and sociability-enhanced TV. Building upon the convergence between different technological infrastructures, such as broadcasting, telecommunications, and the Internet, $\mathrm{TV}$-enriched communication has encouraged new ways to communicate in the context of TV. This concept is not to combine communication services simply as "triple play" does but to encourage communication between users through TV interactions. We propose an application named TV Buddy to demonstrate the TV-enriched communication. The great success of social networking shows that user experiences are enriched and enhanced by sharing, tagging and aggregating collaboratively. The goal of sociability-enhanced TV is to improve TV watching experience by the collaborative use of IPTV. In this paper, the service Social EPG is proposed to demonstrate the sociability-enhanced TV. 
The rest of paper is organized as follows. Section II describes the involved key technologies. Section III proposes the new features of Social TV. A prototype system is developed in Section IV. Finally, Section V summarizes the paper and overviews the future work.

\section{KeY TEChNOLOGIES}

There are two key technologies involved in IMS-based social TV: service architecture and multimedia metadata, which are described in below two parts respectively.

\section{A. Service Architecture}

IMS is considered to be the service architecture for Next Generation Network (NGN), offering multimedia services and enabling service convergence based on diverse network access technologies. Besides the fully defined service architecture, IMS provides inbuilt support for security, charging and Quality of Service (QoS) etc., which is desirable for IPTV. The need for standardized framework for IMS-based IPTV has been recognized by the Telecoms and Internet Converged Services and Protocols for Advanced Networks (TISPAN). TISPAN is the technical committee of the European Telecommunication Standards Institute (ETSI), who is responsible for the integration of multimedia services in NGN. TISPAN addresses the development of standards for an IPTV system that makes use of the IMS architecture and its features [8]. TISPAN Release 3 also brings value-added and blended IPTV service.

With the advantage of an All-over-IP network, the opportunity for integration and convergence is amplified in IMS. The IMS service framework can be used to implement new services, but its main strength might eventually be in its ability to seamlessly integrate existing services and deliver them to end-users in an optimized manner [9]. Considerable standardization activities have been conducted to define independent and self-contained service building blocks, which are known as service capabilities $[10,11]$ in the Third Generation Partnership Project (3GPP) or service enablers [12, 13] in the Open Mobile Alliance (OMA). Thanks to this modular approach, the service blocks can be used independently or reused by different applications. In this paper, we leverage the advantage capabilities of IMS to implement the concept of social TV.

\section{B. Multimedia Metadata}

Metadata is structured information that describes, explains, locates or otherwise makes it easier to retrieve, use or manage an information resource. The interoperable issue of IPTV metadata has been widely recognized by different Standard Development Organizations (SDOs), such as Digital Video Broadcasting (DVB), TV-Anytime (TVA), and Open IPTV Forum (OITF). DVB was the first SDO worldwide with a full set of standards for IPTV, which offer a good foundation for the delivery of IPTV and have been chosen by other SDOs. Within the domain of IPTV, there exist two types of metadata: Service Discovery \& Selection (SD\&S) and Broadband Content Guide (BCG). SD\&S metadata is a set of data related to the SD\&S mechanism as defined by DVB [14]. SD\&S provides for the discovery and selection of all available services for each service provider, such as broadcasting services, VoD services, BCG services, and so on. BCG is a set of data related to the description of pieces of contents and services. In BCG, there are two essential types of information, Program Location Table and Program Information Table [15]. The former table provides the location information where a program is located indirectly by Content Reference Identifier (CRID) [16]. The CRID Location Resolution is the process of mapping a CRID to other CRIDs (e.g., for a series) or to a locator (e.g., the request URL). A locator provides accurate location and timing information for where and when to retrieve the content. The latter table provides the program description which includes title, synopsis and genre etc. These two kinds of metadata, especially BCG, are intensively used by the application of Electronic Program Guide (EPG) which is an on-screen guide to TV programs allowing a viewer to navigate and select program.

Although IPTV metadata has been widely specified by different SDOs, there are still two issues not well addressed: cross-domain and social phenomena. The concept of cross-domain can be described as "providing TV, Internet, home networks and telecom services as an integrated part of the user TV session" [17, 18]. However, since video contents in the past have been provided either by broadcasters or by optical discs, the IPTV metadata specifications focus on the TV domain only. Secondly, the social phenomena behind watching TV are ignored, such as TV sharing, user-generated content, and collaborative media aggregation etc. So far, there have been no related parts in the current IPTV metadata specifications. To meet above requirements, this paper introduces the concept of CAM4Home (Collaborative Aggregated Multimedia for Digital Home) [19]. CAM4Home is an ITEA-2 project, which focuses on specifying an open metadata framework. Its novelty consists in the ability to encapsulate existing metadata technologies for multiple types of content and also able to incorporate references to content related services. On the basis of CAM4Home model, semantic-coherent entities can be further aggregated into a bundle. Most prominently, the CAM4Home metadata framework defines some prosperities of social metadata including social tag, user comment, and user rating to enrich metadata description collaboratively.

We make use of CAM4Home metadata to represent an IPTV resource as CAM Element where the resource is located by the attribute "essenceFileIdentifier" which is a Universal Resource Locator (URL). In this paper, we hereafter refer an IPTV resource to a program or a channel. The IPTV resource is described by CAM Element Metadata within which BCG metadata and social metadata can be associated. CAM Object is the integrated representation of CAM Element and CAM Element Metadata on the association rule "isMetadataOf". Fig. 1 illustrates the CAM Object. CAM Bundles are the aggregation of two or more CAM Objects on the association rule "containsCAMObjectReference". The CAM4Home metadata model supports the representation of a wide variety of media content and service in CAM Element as well as its 
descriptive metadata. Consequently, media in different domains can be aggregated with IPTV on the metadata model. To be noted, we do not try to replace IPTV metadata standards by CAM4Home but to use the latter as a self-defined container. We argue that this approach can not only provide the support of cross-domain but also enrich the social interaction on TV. The use of CAM4Home metadata will be further described in the next section.

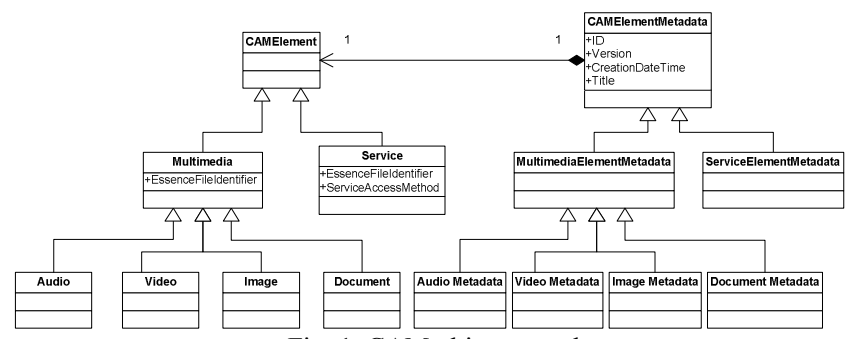

Fig. 1. CAM object metadata

\section{IMS-BASED SOCIAL TV}

In this section, we present the social TV from two aspects respectively: the TV-enriched communication and sociability-enhanced TV.

\section{A. TV-enriched Communication}

$\mathrm{TV}$-enriched communication has encouraged new ways to communicate in the context of TV. The integration of communication services and IPTV could allow users to interact more actively. For example, IPTV integrated with Presence and Instant Messaging (IM) would allow users not only to see if their friends are online, but also to chat or share information about the programs that they are watching on TV. We develop an application named TV Buddy to demonstrate the TV-enriched communication. TV Buddy can be seen as an application of messaging service, aiming to build communities for TV users to communicate with each other and share TV experiences. Distinguished from the traditional IM clients, our TV Buddy is extended in two ways. Firstly, the BCG metadata is integrated into TV Buddy by which users can know what TV programs their friends are watching and switch to them conveniently. Secondly, the users can share IPTV resources to their friends by TV Buddy just like sending a message or sharing a file. It can be utilized by users to invite their friends to watch the same program and even make comments by message or emotion icons to express their feelings.

Based on the IMS service architecture, TV Buddy is implemented by integrating two OMA enablers: IM and Presence in the context of IPTV. The messaging service is a fundamental part of communication today. IMS takes the messaging to a new converged multimedia experience, which allows end users to exchange text, image, video, and even application-specific data. The Message Session Relay Protocol (MSRP) [20] is proposed for transmitting a series of related instant messages in the context of a session, which can be used for file sharing. Presence is a dynamic profile of status information of people, application, or machines that is visible to others. The presence feature enables a user to store, manage, and publish presence information that can be viewed by authorized users. The Presence Information Data Format (PIDF) [21] defines the basic XML format for presenting presence information.

TV sharing is implemented by transmitting CAM metadata entities through IM. As mentioned in Section II, an IPTV resource is encapsulated as CAM Object. Specifically, CAM Element Metadata is utilized for program description information, while CAM Element is for program location information. Regarding the metadata generation, the CAM4Home project provides a web service platform for metadata generating, validating, and registering. The original metadata generated by the service platform are mainly used to describe the IPTV resources according to the basic BCG metadata including title, description and CRID etc. Meanwhile, users are allowed to add their tags, comments or ratings during metadata generation. In order to share CAM4Home metadata entities, we propose two ways. The first one indirectly makes use of CAM identifier. After being registered, each CAM Object can be uniquely identified by "camElementID" in the centralized CAM4Home service platform. So this identifier can be shared between users. Alternatively, CAM entities are transferred directly by IM file sharing. The MIME type "application/cam $x$ ml" for CAM4Home metadata is proposed to register in Internet Assigned Number Authority (IANA). The former method extension is light-weight in term of the verbose XML data. However, the receiver has to access CAM4Home service platform to retrieve the metadata after getting "camElementID". It is worth noting the rich description of metadata may help the receiver to accept or refuse the shared resources.

In terms of Presence, OITF has extended the PIDF schema [21] by adding BCG metadata that declares the current watching TV program information. The present schema extension for OITF Presence service is defined in [22]. In the extended XML schema, each service is described by the "service-description" parameter. A new "service-id" is defined for IPTV with the following values: IPTV-SC (Scheduled Content), IPTV-CoD (Content on Demand), IPTV-NPVR (Network PVR), IPTV-Hybrid (DVB-T/H/C/S). When the user has an active IPTV Presence service, the <tuple> element pertaining to the active service contains the corresponding element. However, the extended schema by OITF is not efficient enough. For example, both serviceID and programID are defined to identify a SC program. In that case, the watcher has to access BCG service again in order to get the names of service and program every time when the publisher changes a program. Therefore, we propose to add the program describing attributes (e.g., service name and program name) directly. Fig. 2 presents an example of our PIDF extension.

\footnotetext{
$<$ ?xml version $=" 1.0 "$ encoding $=" U T F-8 " ?>$

$<$ presence xmlns:xsi="http://www.w3.org/2001/XMLSchema-instance" $x m \ln :$ :iptv $=$ "urn:oipf:service:oitfpresence:2008"

xmlns:oma="urn:oma:xml:prs:pidf:oma-pres"

xmlns="urn:ietf:params:xml:ns:pidf" entity="sip:someone@example.com">

$<$ import schemaLocation ="service-presence.xsd" />

$<$ tuple id="5715603041256833982">

$<$ status $>$
} 


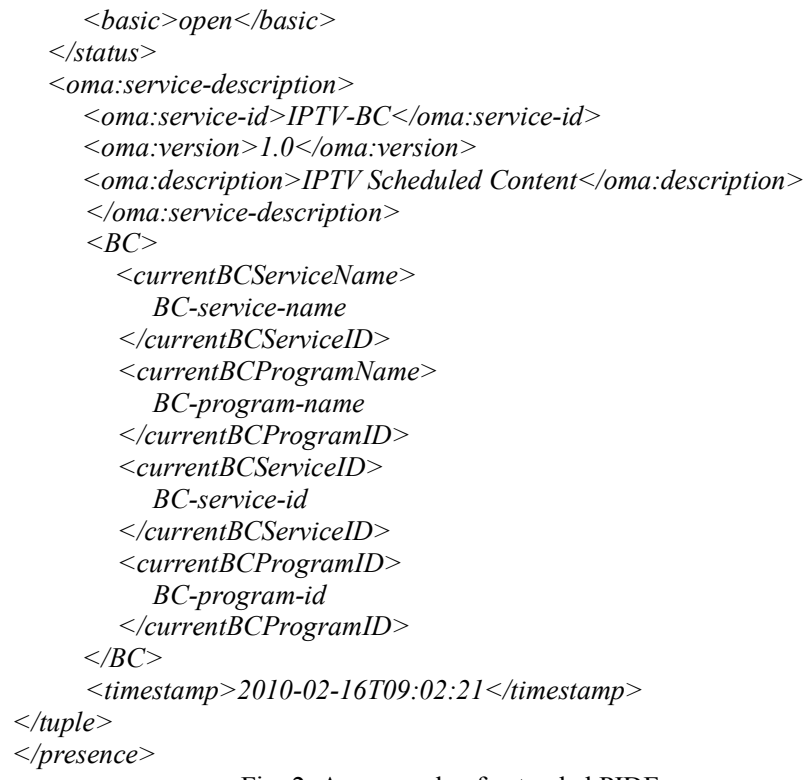

Fig. 2. An example of extended PIDF

\section{B. Sociability-enhanced TV}

Traditionally, the metadata of SD\&S and BCG originated from service providers or content providers are intensively utilized by the well-known application of EPG. However, the lack of metadata is becoming a barrier for the in-depth study and wide application. On the other hand, viewers are exposed to the expansion of TV programs in the digital era. In order to overcome the information overload caused by the growing number of programs, considerable researches have been conducted to develop personalized EPG [23]. However, the process of human decision is driven not only by personal preferences but also by social relationships [24]. Recently, the great success of social networking has shown that user experiences are enriched by collaborating such as sharing, aggregating, and tagging. Under this trend, the social metadata are worth being explored to enhance TV experiences. Social metadata is data generated by collaborative tagging, such as tags, ratings, and comments etc., added to content by individual users other than content providers or service providers. Examples can be found everywhere on the web, ratings and comments on YouTube, tagging in Delicious. The social metadata can help users navigate to relevant contents even quicker because members can use them to provide context and relevant description to the content.

In this paper, we propose the application named Social EPG to demonstrate the concept of sociability-enhanced TV. The Social EPG is an extended version of the traditional EPG, which allows users to share, save, organize, and search TV resources, typically in the form of tags, ratings and comments. Specifically, the traditional EPG is extended from two aspects. At first, the Social EPG supports to share the watching IPTV resource or any resource listed in the EPG. Secondly, it is able to retrieve and show the shared resources from friends as well as the related social metadata along with the tradition EPG metadata. Parallel with the IPTV service, Social EPG is implemented with the convergence of telecom and Internet. Many popular social networking websites are providing a set of
Application Programming Interfaces (APIs) for the development of standalone application. For instance, the Facebook Platform [25] provides a framework for the third party developer to create applications that interact with core Facebook features. In terms of implementation, we take Facebook as an example. The Facebook APIs use a Representational State Transfer (REST) interface over HTTP GET or POST requests. Via the REST-like interface, a Facebook user needs to set some necessary arguments, such as URL, title, description, and tags (optional) etc in order to share a resource. In our solution, the CRID is set as the URL of an IPTV resource. Both title and description are extracted from BCG metadata of the specific IPTV item without user intervention. Through the Facebook APIs, the Social EPG client can publish an IPTV resource. Moreover, it can retrieve the shared resources from the friend list of user.

\section{PROTOTYPE SYSTEM}

We develop a prototype system to demonstrate the IMS-based social IPTV service, which is illustrated in Fig. 3. The prototype is built on the top of Ericsson Software Development Studio (SDS) [27]. The Ericsson SDS provides an end-to-end simulation environment for the design, development and test of value-added services that leverage the capabilities of IMS. SDS can be configured as an IMS core network emulator and also as a cost-effective service execution environment. Service enablers such as IMS Messaging (IMS-M), Presence and Group Management (PGM) as well as an end-to-end IPTV are supported and integrated in SDS. The IPTV emulator supports IPTV developers by running a middleware that simulates and enables IMS-based IPTV applications. SDS also provides a client simulator, namely IMS Client Platform (ICP).

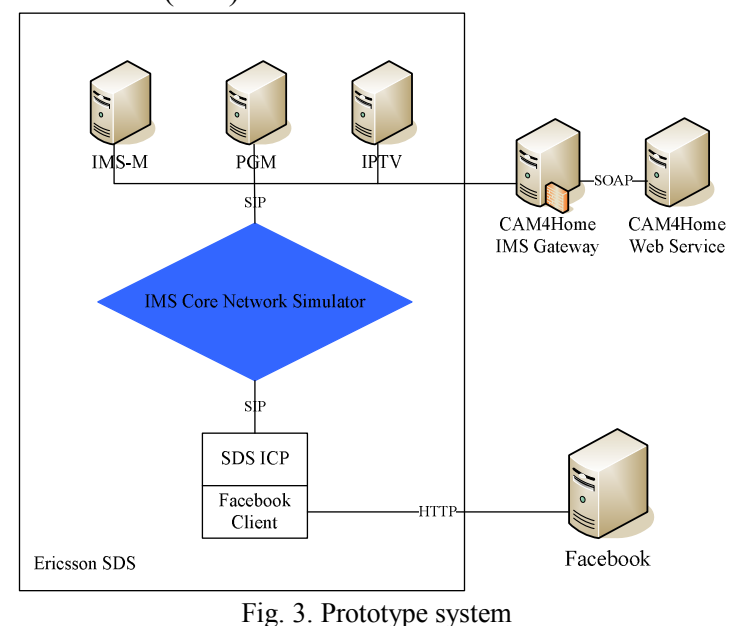

In order to enable the IPTV client to access the CAM4Home MP Web service, we deploy a gateway between IMS and CAM4Home. For instance, the basic BCG metadata including title, description and CRID etc. are extracted by the client of IPTV emulator and sent to the gateway for generating metadata. The IMS gateway is set up by SUN GlassFish Servlet [27] and configured to connect with Ericsson SDS. Furthermore, a Facebook client [28] is integrated for the social 
extension of EPG. The initial tests show that the proposed extensions work compatibly with the standardized IMS platform. The initial tests have verified all functions proposed in Section III. Currently, both the Web service client and the Facebook client work separately from the SDS ICP. We are working on an integrated client.

\section{CONCLUSION}

We have proposed a novel application named Social TV over IMS infrastructure. The traditional IPTV service is extended to bundle with existing communications services such as IM and Presence. On the other hand, the IPTV service is converged with Internet to be enhanced with sociality. Based on the advantage of IMS service architecture, we develop a prototype system to demonstrate our proposed extensions. The further work is expected to improve the usability and combine separated functionalities.

\section{ACKNOWLEDGMENT}

This work was supported by the ITEA-2 CAM4Home project. We would like to thank all partners for their contributions.

\section{REFERENCES}

[1] A. Takahashi, D. Hands and V. Barriac, "Standardization Activities in the ITU for a QoE Assessment of IPTV", IEEE Communications Magazine, vol. 46, no. 2, pp. 78-84, Feb. 2008.

[2] 3GPP TS 23.228. IP Multimedia Subsystem (IMS), stage 2.

[3] B. Chatras, M. Said, "Delivering Quadruple Play with IPTV over IMS", the International Conference on Intelligence in Networks (ICIN), 2007.

[4] T. Coppens, L. Trappeniers, and M. Godon, "AmigoTV: Towards a Social TV Experience", The 2nd Europe Conference on Interactive TV, 2004.

[5] M. Nathan, C. Harrison, S. Yarosh, L. Terveen, L. Stead, and B. Amento, "CollaboraTV - Making TV Social Again", The 1st International Conference on Designing Interactive User Experiences for TV and Video, 2008.

[6] E. Boertjes, "ConnecTV: Share the Experience", European Conference on Interactive TV, 2007.

[7] B. Falchuk, K. Sindar, S. Loeb, and A. Dutta, "Mobile Contextual Mashup Service for IMS", The $2^{\text {nd }}$ IEEE International Conference on Internet Multimedia Services Architecture and Application, Bangalore, Dec. 2008.

[8] ETSI TS 182027 V2.0.0, TISPAN, "IPTV Architecture; IPTV Functions Supported by the IMS Subsystem", Feb. 2008.

[9] Gourraud. C., "Using IMS as a Service Framework", IEEE Vehicular Technology Magazine, vol. 2, pp: 4-11, Mar. 2007.

[10] 3GPP TS 24.141. Presence service using the IP Multimedia (IM) Core Network $(\mathrm{CN})$ subsystem.

[11] 3GPP TS 24.247. Messaging using the IP Multimedia (IM) Core Network (CN) subsystem.

[12] OMA Presence SIMPLE V2.0, "Architecture Document”, Dec. 2008.

[13] OMA SIMPLE IM V1.0, "Instant Messaging using SIMPLE Architecture", Sep. 2008.

[14] ETSI TS 102034 V1.4.1, "Digital Video Broadcasting (DVB); Transport of MPEG-2 TS Based DVB Services over IP Based Networks", Aug. 2009.

[15] ETSI TS 102539 V1.2.1, "Digital Video Broadcasting (DVB); Carriage of Broadband Content Guide (BCG) Information over Internet Protocol (IP)", Apr. 2008.

[16] Open IPTV Forum, "Release 1 Specification Volume 3 - Content Metadata V1.1", Oct. 2009.
[17] M. Guelbahar, J. Zoric, "Service and Content Metadata Systems in Interactive TV Domains", The $1^{\text {st }}$ International ICST Conference on User Centric Media, Dec. 2009.

[18] C. Hesselman, W. Derks, J. Broekens, H. Eertink, M. Guilbahar, R. Poortinga, "An Open Service Infrastructure for Enhancing Interactive TV Experiences", European Conference on Interactive TV, 2008.

[19] CAM4Home Official Website. Available: http://www.cam4home-itea.org/.

[20] IETF RFC 4975, "The Message Session Relay Protocol (MSRP)", Sep. 2007.

[21] IETF RFC 3863, "Presence Information Data Format (PIDF)", Aug. 2004.

[22] Open IPTV Forum, "Release 1 Specification Volume 4 - Protocols V1.1", October 2009.

[23] H. Zhang, S. Zheng, and J. Yuan, "A Personalized TV Guide System Compliant with MHP", IEEE Transactions on Consumer Electronics, Vol. 51, No. 2, pp. 731-737, 2005.

[24] A. Iatrino, and S. Modeo, "EPG-Board a Social Application for the OmegaBox Media Center", European Conference on Interactive TV 2007.

[25] Facebook Developer. Available: http://developers.facebook.com/,

[26] Ericsson Service Development Studio (SDS) 4.2. Available: http://www.ericsson.com/developer/sub/open/technologies/ims_poc/tool s/sds 40 .

[27] SUN GlassFish Servlet. Available: https://glassfish.dev.java.net/.

[28] Facebook Java API Client. Available: http://code.google.com/p/facebook-java-api/. 